\title{
Conductive atomic force microscopy as a tool to reveal high ionising dose effects on ultra thin $\mathrm{SiO}_{2} / \mathrm{Si}$ structures
}

\author{
Richard Arinero $\cdot$ A. D. Touboul $\cdot$ M. Ramonda • \\ C. Guasch • Y. Gonzalez-Velo • J. Boch • \\ F. Saigné
}

Received: 18 March 2012/Accepted: 17 April 2012/Published online: 5 May 2012

(C) The Author(s) 2012. This article is published with open access at Springerlink.com

\begin{abstract}
The electrical stress behaviour of non-irradiated and irradiated ( 2 to $7-\mathrm{nm}$ thick) $\mathrm{SiO}_{2} / \mathrm{Si}$ structures is investigated using conductive-atomic force microscopy. A protocol based on the successive application of two rampedvoltage stresses (RVS) on each test point is performed. The environmental implementation conditions of such an experiment are then investigated. A statistical approach based on the use of Weibull distributions is also adopted. Before irradiation, for the thinnest samples, it is shown evidence of stress-induced trap-assisted tunnelling leading to a high decrease in threshold voltages on the second RVS. After high-dose X-ray irradiations, the first RVS exhibit voltage-shift effects increasing with the oxide film thickness, whereas for the second RVS, no additional effect is observed. The high locality of these measurements, sensitive to a few tens of trapped charges, is therefore demonstrated and constitutes a new step towards a better understanding of oxide degradation mechanisms due to radiation effects.
\end{abstract}

Keywords Conductive-AFM (C-AFM) - Weibull statistic · Ramped voltage stress $\cdot$ Nano I-V curves $\cdot$ Ultra-thin oxide films $\cdot \mathrm{SiO}_{2} / \mathrm{Si}$ structures $\cdot$ Oxide reliability

R. Arinero $(\bowtie) \cdot$ A. D. Touboul · C. Guasch ·

Y. Gonzalez-Velo · J. Boch · F. Saigné

Institut d'Electronique du Sud (IES), Université Montpellier 2, UMR CNRS 5214, CC083, Place Eugène Bataillon,

34095 Montpellier Cedex, France

e-mail: richard.arinero@univ-montp2.fr;

richard.arinero@ies.univ-montp2.fr

\section{Ramonda}

Laboratoire de Microscopie en Champ Proche,

Université Montpellier 2, CC082, Place Eugène Bataillon,

34095 Montpellier Cedex, France

\section{Introduction}

Degradation and breakdown of gate oxide is a crucial issue for the reliability of MOS devices. Conventional tests are usually made at the device scale, although it has been shown that breakdown can be a highly localised phenomenon (DiMaria and Stathis 1997). Advances in our ability to explore local properties make possible to gain a deeper understanding of basic mechanisms involved. In this context, it is important to use reliable methods for the determination of electric properties at nanoscale. Conductiveatomic force microscopy (C-AFM) is an AFM-based current sensing technique allowing producing $\mathrm{I}-\mathrm{V}$ curves in the range of hundreds of $\mathrm{fA}$ to a few $\mu \mathrm{A}$ with nanometer spatial resolutions (Fig. 1). C-AFM can simultaneously map topography and current distributions of a sample. Numerous studies have been realised on thin $\mathrm{SiO}_{2}$ films on Si substrates using this technique (Porti et al. 2002, 2003, 2007; Wu and Lin 2006; Wu et al. 2007, 2008; Hourani et al. 2011). But, at this day, no clear explanation was provided to understand the mechanisms involved at nanoscale in stressed and broken-down thin films.

The most accomplished works can be attributed to Porti et al. $(2002,2003,2007)$ and $\mathrm{Wu}$ et al. $(2007,2008$; Wu and Lin 2006). Both authors have shown that oxide degradation exhibits two aspects. First, an electrical aspect where an important leakage current is measured after the application of an electrical stress. Second, a physical aspect corresponding to the formation of nano-sized "hillocks" at the oxide's surface. We note that the presence of these hillocks is very surprising, because it is observed although the substrate is biased negatively and the tip positively, that is to say, when no anodic oxidation is expected at the surface. According to Hourani et al. (2011), hillocks could be caused by two phenomena: a real modification of the 


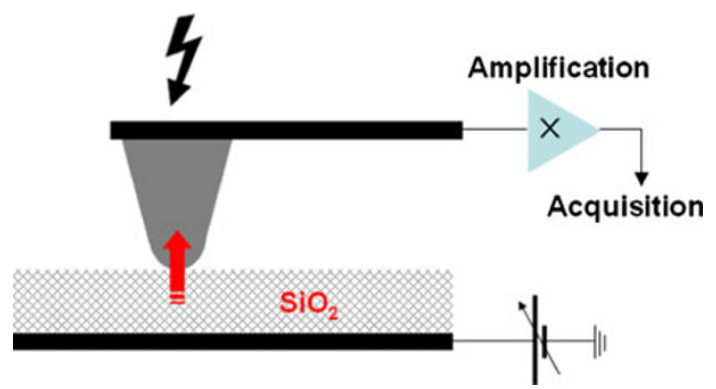

Fig. 1 Principle of conductive atomic force microscopy (C-AFM). The current flowing through the oxide can be measured in the range of hundreds of fA to a few $\mu \mathrm{A}$

oxide's topography and an electrostatic interaction between trapped charges at the oxide/substrate interface and the AFM tip. The size of one hillock increases with the stress time (i.e., the quantity of charges passing through the oxide). One of the common points of all these studies is that statistical approaches have rarely been carried out, although it is admitted that breakdown is a statistical process. Wu et al. (2008) are the only ones to have reported time-to-breakdown distributions using a Weibull representation. Concerning C-AFM studies on irradiated oxides, we can make reference to the results published by Porti et al. (2007) in which they give evidence of nano-sized leaky spots due to heavy-ions impacts on very thin $\mathrm{SiO}_{2}$ gate oxides. With respect to ionising dose effects, very little was reported in the literature. Wu et al. (2007) have observed bias-annealing effects in $\mathrm{Co}^{60} \gamma$-ray irradiated very thin $\mathrm{SiO}_{2}$ films.

In this paper, we report on $1 \mathrm{Mrad} \mathrm{X}$-ray irradiated ultrathin $\mathrm{SiO}_{2} / \mathrm{n}-\mathrm{Si}$ structures studied by C-AFM. Statistical results of threshold voltages are presented in comparison with non-irradiated structures. The threshold voltage is defined as the voltage necessary to reach a few pA leakage current through the oxide film. The conditions of acquisition of reproducible ramped-voltage stress curves are first discussed, based on non-irradiated samples observations, and then achieved to study irradiated samples of different thicknesses.

\section{Experimental procedure}

Test structures consist in thermal 2, 4 and $7-\mathrm{nm} \mathrm{SiO}_{2}$ oxides grown on p-type $\left(\sim 10^{18} \mathrm{~cm}^{-3}\right) \mathrm{Si}$ substrates provided by the CEA leti-MINATEC, Grenoble (France) Technology platform. C-AFM experiments have been performed using a Veeco Dimension $5^{\mathrm{TM}}$ AFM equipped with a Nanoscope $5^{\mathrm{TM}}$ controller and a TUNA ${ }^{\mathrm{TM}}$-extended module. The AFM head is placed in a vacuum chamber at a pressure of $\sim 10^{-5}$ mbar. In the imaging configuration, topography and current images are acquired in the contact mode whilst a constant bias is applied between the tip and the sample. In the spectroscopy configuration, I-V curves are collected at a local fixed position. We used PtCr-coated AFM tips (Budget Sensors ${ }^{\mathrm{TM}}$ ) having a typical cantilever stiffness $k_{\mathrm{c}}=2.8 \mathrm{~N} \mathrm{~m}^{-1}$ and a curvature radius of about $R=20 \mathrm{~nm}$ for a new tip. The degradation mechanisms of $\mathrm{SiO}_{2}$ films have been studied by means of ramped-voltage stresses (RVS) where the applied voltage is ramped whilst the current flowing through the sample is recorded, leading to I-V curves. During the first ramp, the absolute voltage value increases from 0 to $10 \mathrm{~V}$ with steps of $10 \mathrm{mV}$ at a ramp speed of $1 \mathrm{~V} / \mathrm{s}$. The second ramp corresponds to a backward curve of decreasing absolute voltage from 10 to $0 \mathrm{~V}$. Irradiations were made using a $10-\mathrm{keV} \mathrm{X}$-ray irradiator. A total dose of $1 \mathrm{Mrad}$ was applied to the samples at a rate of $200 \mathrm{rad} / \mathrm{s}$. It is, moreover, important to mention that during the experiments, the substrate was biased negatively and the tip positively, to avoid oxidation at the sample surface. All reported voltages and current values correspond to absolute values.

\section{Results and discussion}

Non-irradiated samples

Typical RVS recorded on 4-nm $\mathrm{SiO}_{2} / \mathrm{p}-\mathrm{Si}$ non-irradiated samples are shown in Fig. 2. Absolute values of the voltage and the current are presented on the curves. The first RVS can be described using a Fowler-Nordheim (FN) tunnelling model where the current starts to increase at a given value of the applied voltage. We can observe that the second RVS is shifted towards lower voltages. This decrease can be explained by the activation of defects generated in the oxide that helps the tunnelling of charges through the

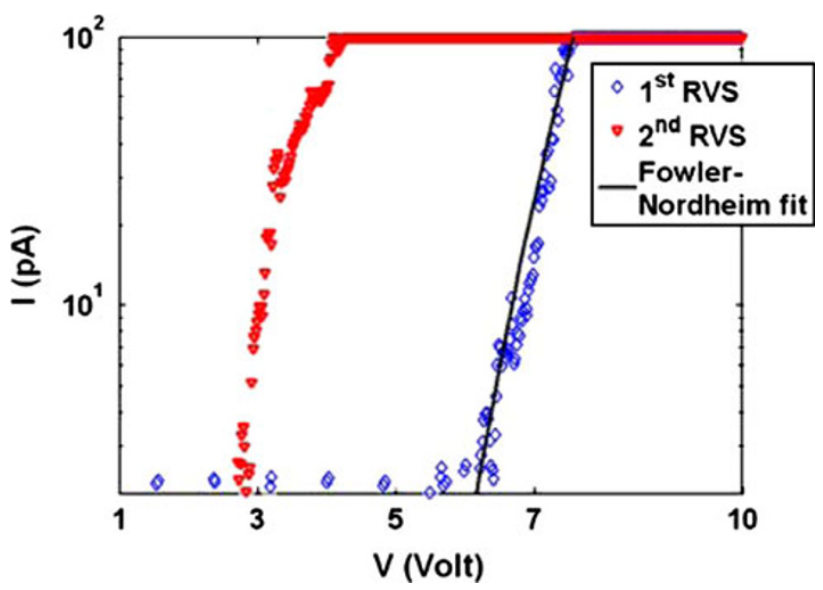

Fig. 2 Typical first and second ramped-voltage stresses (RVS) recorded on non-irradiated $4-\mathrm{nm} \mathrm{SiO}_{2} / \mathrm{p}-\mathrm{Si}$ samples 
oxide, well known as trap-assisted tunnelling (TAT) current (Paskaleva et al. 2008).

We have studied statistical distributions of RVS curves. Using a "point and shoot" function of the instrument, we have performed 25 RVS distributed in a $5 \times 5$ matrix as presented in Fig. 3. This figure shows the topographical modifications obtained at each stressed point. Nano-sized hillocks of about 2-nm height are observed with a good reproducibility as long as the experiments are realised under vacuum. Indeed, we noted that the presence of a water layer in ambient air experiments can lead to a tip oxidation after a few RVS, what is incompatible with statistical studies $\left(\mathrm{Pt}+n \mathrm{H}_{2} \mathrm{O} \rightarrow \mathrm{PtO}_{n}+2 n \mathrm{H}^{+}+2 n \mathrm{e}^{-}\right.$).

The presence of hillocks may be interpreted as a tip/ oxide power and energy dissipation effect. A high local temperature rise up to more than melting point is possible for PtCr tips due to a small contact radius value. Moreover, due to an important oxide layer thermal resistivity, energy is not easily dissipated through the sample and can be stored more long time at the surface.

We define the threshold voltage $V_{\text {th }}$ as the voltage at which the current through the oxide reaches $10 \mathrm{pA}$. The Weibull cumulative distribution function can be arranged in the linear form:

$\ln [-\ln (1-F)]=\beta \ln \left(V_{\text {th }}\right)-\beta \ln \alpha$

in such way that plotting $\ln [-\ln (1-F)]=f\left(\ln \left(V_{\text {th }}\right)\right)$, we get $\beta$ corresponding to the slope of the curve, and $\alpha$ extracted from the ordinate at the origin. $\alpha$ corresponds to the voltage at which $63 \%$ of RVS lead to a $10 \mathrm{pA}$ current and $\beta$ indicates the width of the distribution, i.e., the variability of the measurement. The $V_{\text {th }}$ Weibull distributions of a non irradiated 4-nm $\mathrm{SiO}_{2} / \mathrm{p}$-Si sample are represented in Fig. 4. For the first RVS, we get higher values of $\alpha$ and $\beta$, indicating both higher values and lower variability than

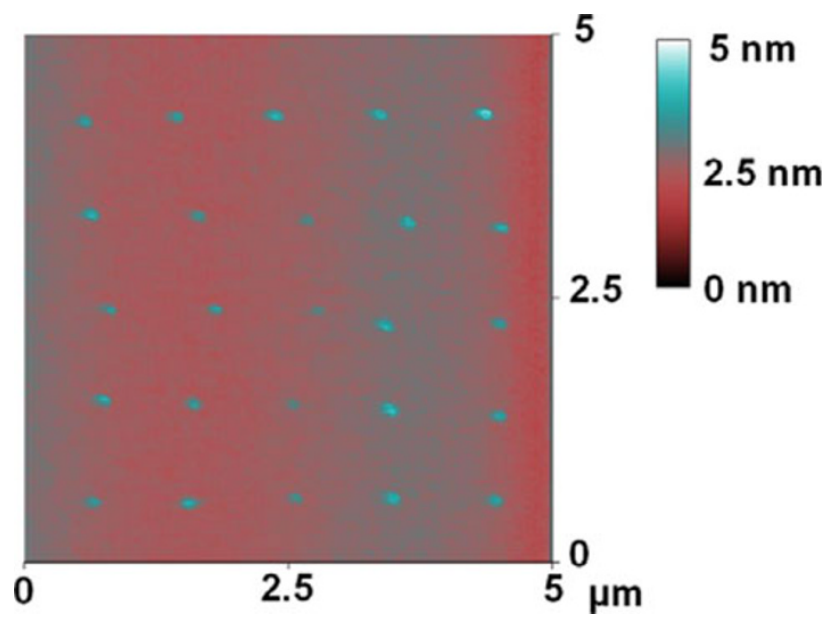

Fig. 3 Stress-induced morphological modifications observed on a non-irradiated $4-\mathrm{nm} \mathrm{SiO}_{2} / \mathrm{p}$-Si sample after $25 \mathrm{RVS}$ realised according to a $5 \times 5$ matrix

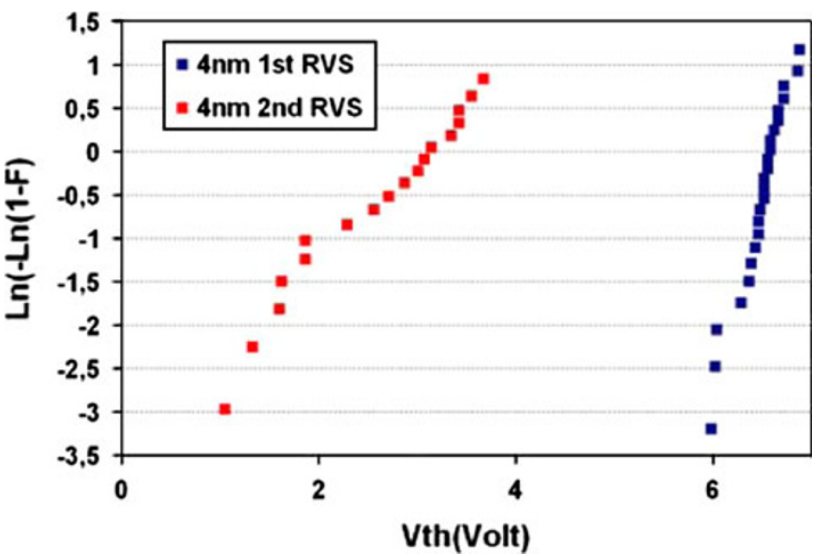

Fig. 425 RVS Weibull distributions of first (blue line) and second (red line) RVS $V_{\text {th }}$ values obtained for a 4-nm thick highly-doped $\mathrm{SiO}_{2} / \mathrm{p}$-Si non-irradiated sample

for the second RVS, in which case, electrical stress is probably responsible for defects generation and TAT.

Table 1 summarises Weibull parameters obtained for different $\mathrm{SiO}_{2}$ thicknesses. Two different trends emerge from these data. The first one is observed for thinner films ( 2 and $4 \mathrm{~nm}$ ) for which $\alpha$ and $\beta$ value are much lower for the second RVS than for the first. The second trend is found for the 7-nm sample for which $\alpha$ is slightly higher for the second RVS than for the first. This effect can be attributed to a small quantity of charges trapped into the oxide. We can thus conclude that TAT phenomenon is observed for thinner samples and no more for thicker samples, probably because, stress-induced traps are not anymore within a tunnelling distance.

\section{Irradiated samples}

Let us now consider $1 \mathrm{Mrad}$ X-ray irradiated samples in comparison with non-irradiated samples. Figure $5 \mathrm{a}, \mathrm{b}, \mathrm{c}$ shows the superposition of 25 first RVS for 2, 4 and 7-nm thick samples, respectively.

For 2 and 7-nm samples, a positive shift of I-V curves is clearly observed after irradiation. For the 4-nm sample, we note a negative shift. The sign of the voltage shift is

Table 1 Weibull parameters $(\alpha$ and $\beta$ ) obtained for the first and second RVS on non-irradiated $\mathrm{SiO}_{2} / \mathrm{p}-\mathrm{Si}$ samples

\begin{tabular}{|c|c|c|c|}
\hline $\mathrm{SiO}_{2}$ film thickness (nm) & 2 & 4 & 7 \\
\hline \multicolumn{4}{|l|}{ First RVS } \\
\hline$\alpha(\mathrm{V})$ & 4.5 & 6.6 & 8.7 \\
\hline$\beta$ & 28.8 & 29.7 & 37.9 \\
\hline \multicolumn{4}{|l|}{ Second RVS } \\
\hline$\alpha(\mathrm{V})$ & 1.96 & 2.6 & 9.3 \\
\hline$\beta$ & 1.12 & 1.44 & 41.65 \\
\hline
\end{tabular}



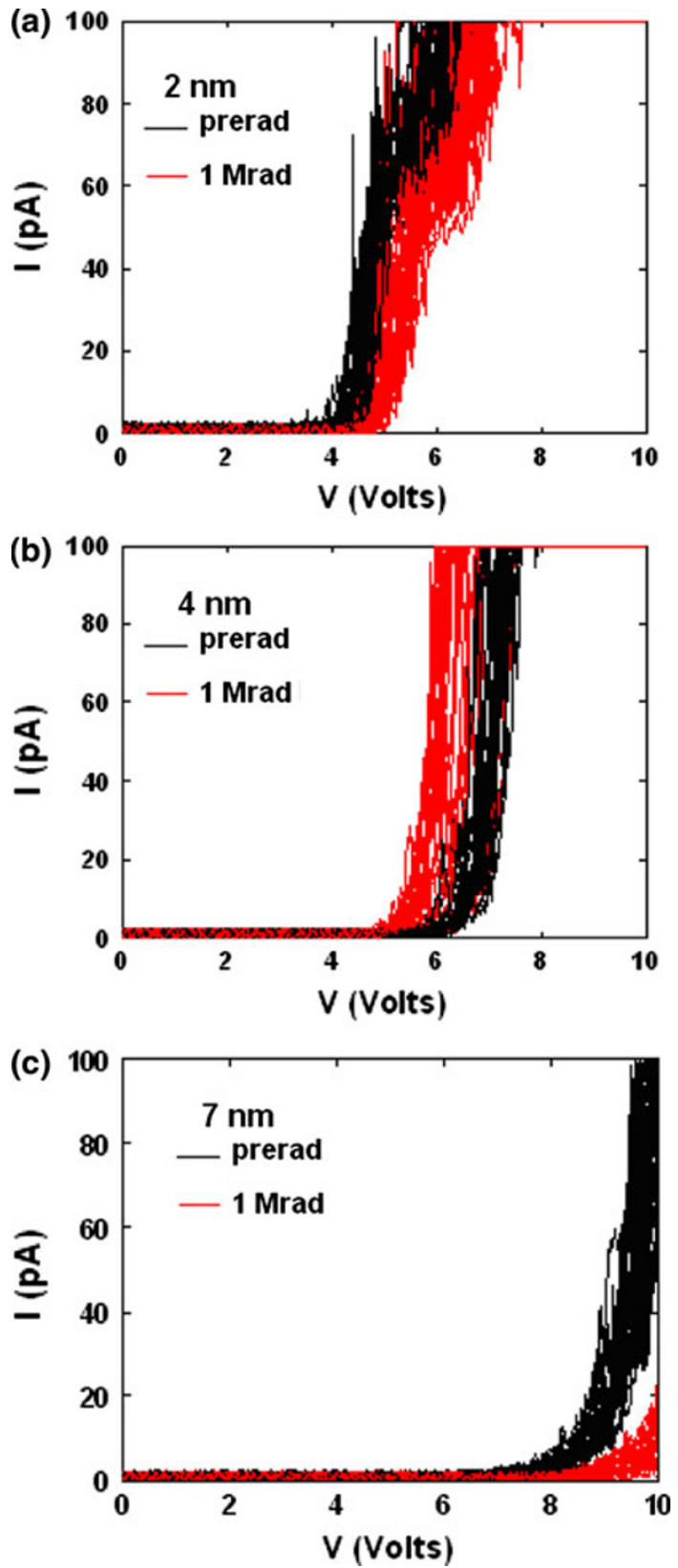

Fig. 525 first RVS recorded on prerad (black line) and on 1 Mrad irradiated (red line) samples of different oxide thickness: a $2 \mathrm{~nm}$, b $4 \mathrm{~nm}$ and c $7 \mathrm{~nm}$ highly doped $\mathrm{SiO}_{2} / \mathrm{p}$-Si samples

difficult to explain. It is probably the resultant of a competition between trapped charges into the oxide and the $\mathrm{SiO}_{2} / \mathrm{Si}$ interface which may be of different sign. Those results have been presented as Weibull plots in Fig. 6 to better identify the characteristics of each degradation profile. For the 2-nm sample, it was found $\alpha=4.54 \mathrm{~V}$ and $\beta=28.8$ before irradiation and $\alpha=5.03 \mathrm{~V}$ and $\beta=36.15$ after irradiation. We obtain almost the same variability and a positive shift $\Delta \alpha$ of about $0.5 \mathrm{~V}$. The $4-\mathrm{nm}$ sample exhibits a negative shift $\Delta \alpha$ of about $0.9 \mathrm{~V}$ and a quite similar low variability $(\alpha=6.61 \mathrm{~V}, \beta=29.7$ for the

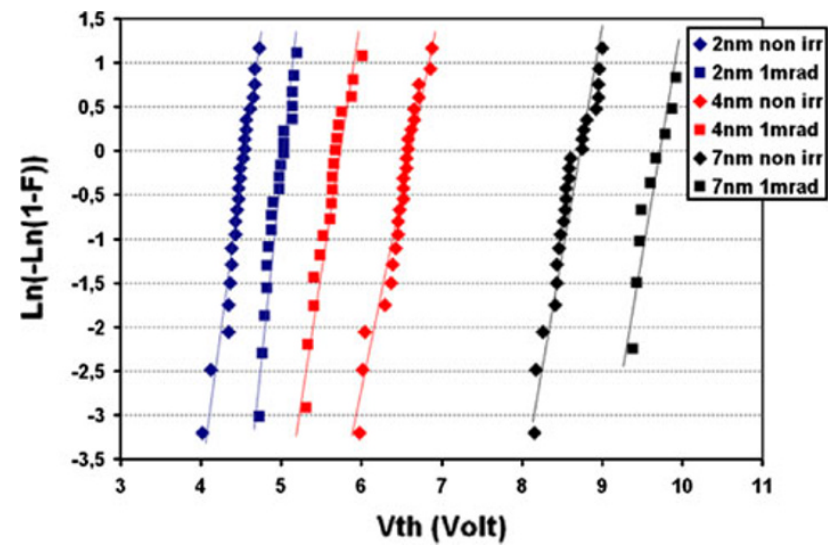

Fig. 6 Weibull plots of first RVS $V_{\text {th }}$ values obtained for nonirradiated (rhombus symbol) and $1 \mathrm{Mrad}$ irradiated (square symbol) $\mathrm{SiO}_{2} / \mathrm{p}$-Si samples at different oxide thickness: $2 \mathrm{~nm}$ (blue line), $4 \mathrm{~nm}$ (red line) and $7 \mathrm{~nm}$ (black line)

prerad measurement and $\alpha=5.72 \mathrm{~V}, \beta=30.4$ after $1 \mathrm{Mrad}$ irradiations). For the 7-nm sample, a positive voltage shift $\Delta \alpha \sim 1 \mathrm{~V}$ is again observed $(\alpha=8.7 \mathrm{~V}$ and $\beta=37.9$ before irradiation and $\alpha=9.7 \mathrm{~V}$ and $\beta=45.3$ after irradiation). From these observations, we can outline that even if the sign of the voltage shift is not always the same, its absolute value increases with the $\mathrm{SiO}_{2}$ film thickness.

We can interpret voltage shifts as a density of oxide or $\mathrm{SiO}_{2} / \mathrm{Si}$ interface trapped charges leading to a modification of the oxide electric field. For instance, because we are unable to differentiate those two types of charges, we only assume a global density of charges $\Delta \mathrm{N}$ with a centroid located at the $\mathrm{SiO}_{2} / \mathrm{Si}$ interface. This is a simple first approach aiming to understand what mechanisms really influence $V_{\text {th }}$ shifts and to estimate the order of magnitude of trapped charges densities.

The FN tunnelling current density is given by Gupta et al. (1997):

$J_{\mathrm{FN}}=C E_{\mathrm{ox}}^{2} \exp \left(-\chi / E_{\mathrm{ox}}\right)$

where $E_{\mathrm{ox}}$ is the electric field into the oxide and $\mathrm{C}$ and $\chi$ are constants depending on the oxide characteristics, equals to $C=9.92 \times 10^{-7} \mathrm{~A} \mathrm{~V}^{-2}$ and $\chi=2.635 \times 10^{8} \mathrm{~V}$ for $\mathrm{SiO}_{2}$ (Gupta et al. 1997). $E_{\mathrm{ox}}$ is given by $E_{\mathrm{ox}}=V_{\mathrm{ox}} / T_{\mathrm{ox}}$ where $V_{\mathrm{ox}}$ is the effective voltage in the oxide and $T_{\mathrm{ox}}$ is the oxide thickness. $V_{\text {ox }}$ can be related to the density of trapped charges $\Delta N$ using the following relation (Gupta et al. 1997):

$V_{\mathrm{ox}}=V_{\mathrm{app}}-\frac{\Delta N \times q}{C_{\mathrm{ox}}}-V_{\mathrm{p}}$

where $C_{\mathrm{ox}}$ is the oxide capacitance per unit of area, $V_{\mathrm{app}}$ is the applied voltage and $V_{\mathrm{p}}$ is the voltage drop in the substrate (the maximum possible value is the $\mathrm{Si}$ band gap $1.12 \mathrm{~V}$ at $300 \mathrm{~K})$. Using expressions (2) and (3), we have 


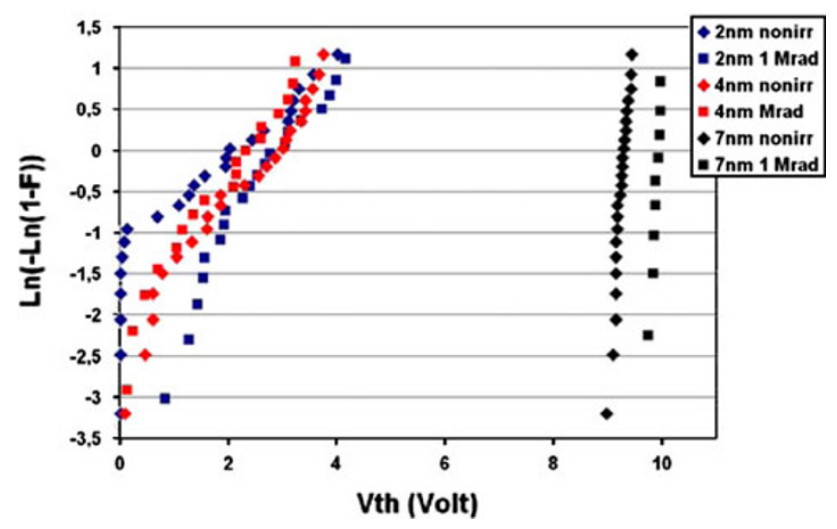

Fig. 7 Weibull plots of second RVS $V_{\text {th }}$ values obtained for nonirradiated (rhombus symbol) and $1 \mathrm{Mrad}$ irradiated (square symbol) $\mathrm{SiO}_{2} / \mathrm{p}$-Si samples at different oxide thickness

estimated the relative increase in net charge, $\Delta N$, meaning without any discrimination between interface and oxide contributions. Calculated values are ranged from $2 \times 10^{12} \mathrm{~cm}^{-2}$ for 2-nm samples to $4 \times 10^{12} \mathrm{~cm}^{-2}$ for 4-nm samples. The contact area of the AFM tip and the oxide's surface being estimated to about $500 \mathrm{~nm}^{2}$, we can then deduce that the quantity of trapped charges probed by the AFM tip is approximately $10-20$ charges. This value demonstrates the high locality of the measurement, and is in good agreement with the number of charges detectable using an atomic force microscope (Dianoux 2004).

Weibull plots of the second RVS of irradiated samples have been represented on Fig. 7 in comparison with those of non-irradiated samples. Both types of samples exhibit very similar behaviours. We note, nevertheless, a nonnegligible voltage shift towards higher values for the 7-nm sample. We can thus conclude that high-dose X-ray irradiations have not a critical incidence on the stress-induced degradation of very thin $\mathrm{SiO}_{2}$ lower than $7 \mathrm{~nm}$. At the opposite, as shown previously, high-dose X-ray irradiations seem to have more important effects on the first RVS behaviour of samples lower than $7 \mathrm{~nm}$.

\section{Conclusion}

We have investigated the electrical stress behaviour of nonirradiated and X-ray irradiated highly doped $\mathrm{SiO}_{2} / \mathrm{p}-\mathrm{Si}$ structures at the nanoscale by means of C-AFM. A statistical approach based on the use of Weibull distributions has been adopted. We have shown that the oxide degradation due to the stress leads to a high decrease of the threshold voltage of I-V curves. This decrease corresponds to a trapassisted tunnelling effect. On the other hand, we have observed, for the first time at the nanoscale, that $1 \mathrm{Mrad}$ $\mathrm{X}$-ray total dose exposure impacts the first RVS curves by important threshold voltages shifts. Different shift signs have been observed on samples of variable thickness. Moreover, we have noted that shifts absolute values increase with the sample thickness. For instance, we cannot explain this effect otherwise than by a competition between trapped charges of opposite sign, either in the oxide or at the $\mathrm{SiO}_{2} / \mathrm{Si}$ interface, modifying the electric field into the oxide layer. On the other hand, the second RVS of thinnest samples ( 2 and $4 \mathrm{~nm}$ ), representative of oxide degradation, were not impacted more after high-dose X-ray irradiations. For thicker samples $(7 \mathrm{~nm})$, we found slight voltage shifts probably due to charge trapping. We will concentrate our efforts in the near future to allow C-AFM measurements to distinguish the nature and proportion of trapped charges. More precise models are still needed to provide such information. Those prospective results, nevertheless, open the way to further studies on ionising radiation effects at nanoscale using C-AFM experiments, including for example temperature and moisture effects.

Acknowledgments The authors are grateful towards Yveline Gobil and Amal Chabli from the CEA Leti-Minatec technology platform (Grenoble, France) for $\mathrm{SiO}_{2} / \mathrm{Si}$ samples preparation. This work was realized as a part of PiGS project (ANR 2010 BLAN 031401) sponsored by National Agency for Research (France). The financial support of the "Réseau National des Grandes Centrales de Technologies" (RTB-Projet Exogène) is acknowledged.

Open Access This article is distributed under the terms of the Creative Commons Attribution License which permits any use, distribution, and reproduction in any medium, provided the original author(s) and the source are credited.

\section{References}

Dianoux (2004) R, Injection et detection de charges dans des nanostructures semiconductrices par Microscopie à Force Atomique, $\mathrm{PhD}$ thesis, Université Grenoble 1, 2004

DiMaria DJ, Stathis JH (1997) Ultimate limit for defect generation in ultra-thin silicon dioxide. Appl Phys Lett 71(22):3230-3232

Gupta A, Fang P, Song M, Lin MR, Wollesen D, Chen K, Hu C (1997) Accurate determination of ultrathin gate oxide thickness and effective polysilicon doping of CMOS devices. IEEE Electron Device Lett 18(12):580-582

Hourani W, Gautier B, Militaru L, Albertini D, Descamps-Madine A (2011) Study of the physical and electrical degradation of thin oxide by atomic force microscope. J Vac Sci Technol B 29(1):01AA06-1-01AA06-8

Paskaleva A, Yanev V, Rommel M, Lemberger M, Bauer J (2008) Improved insight in charge trapping of high- $\mathrm{ZrO}_{2} / \mathrm{SiO}_{2}$ tracks by use of tunnel atomic force microscopy. J Appl Phys 104(2):024108-1-024108-2

Porti M, Nafria M, Aymerich X (2002) Electrical characterization of stressed and broken down $\mathrm{SiO}_{2}$ films at a nanometer scale using a conductive atomic force microscope. J Appl Phys 91(4): 2071-2079

Porti M, Nafria M, Blum MC, Aymerich X, Sadewasser S (2003) Atomic force microscope topographical artifacts after the 
dielectric breakdown of utltrathin $\mathrm{SiO}_{2}$ films. Surf Sci 532-535: $727-731$

Porti M, Gerardin S, Nafria M, Aymerich X, Cester A, Paccagnella A (2007) Using AFM related techniques for the nanoscale electrical characterization of irradiated ultrathin gate oxides. IEEE Trans Nucl Sci 54(6):1891-1897

Wu YL, Lin ST (2006) Two-trap assisted tunneling model for postbreakdown I-V characteristics in ultrathin silicon dioxide. IEEE Trans Device Mater Reliab 6(1):75-80
Wu YL, Lin ST, Chang TM, Liou JJ (2007) Nanoscale bias-annealing effect in postirradiated thin silicon dioxide films observed by conductive atomic force microscopy. IEEE Trans Device Mater Reliab 7(2):351-355

Wu YL, Lin ST, Lee CP (2008) Time-to-breakdown distribution of thin gate oxide subjected to nanoscaled constant-voltage and constant-current stresses. IEEE Trans Device Mater Reliab 8(2):352-357 